ANNALES

POLONICI MATHEMATICI

$90.3(2007)$

\title{
A new class of pluripolar sets
}

\author{
by Nguyen Quang Dieu and Tang Van Long (Hanoi)
}

\begin{abstract}
Let $D$ be a domain in $\mathbb{C}^{n}$. We introduce a class of pluripolar sets in $D$ which is essentially contained in the class of complete pluripolar sets. An application of this new class to the problem of approximation of holomorphic functions is also given.
\end{abstract}

I. Introduction. In [Sa] Sadullaev studied, among other things, the question of rapid approximation of holomorphic functions by rational functions. One of his main results says that if a closed set $E$ is a countable union of complex hypersurfaces then every holomorphic function on $\mathbb{C}^{n} \backslash E$ can be approximated rapidly and uniformly on compact sets by a sequence of rational functions. It should be mentioned that Sadullaev's method yields a stronger result, namely that the above theorem is still valid if $E$ is complete pluripolar (see Lemma 5 in [Sa]). Here by complete pluripolar, he means a closed set $E$ in $\mathbb{C}^{n}$ with the following properties: for any compact sets $K \subset \mathbb{C}^{n} \backslash E$ and $L \subset E$, there are constants $C, \delta>0$ and a sequence $\left\{p_{m}\right\}$ of polynomials of degree at most $m$ such that $\inf _{K} m^{-1} \log \left|p_{m}\right|>\log \delta$, $\sup _{L} m^{-1} \log \left|p_{m}\right|<-\log m$, and $\sup _{\Delta^{n}}\left|p_{m}\right|=1$, where $\Delta^{n}$ is the unit polydisk in $\mathbb{C}^{n}$. This concept, in higher dimensions, is formally quite different from the usual definition of complete pluripolar set (being the singular locus of some (non-trivial) global plurisubharmonic function). However, in one dimension, every closed polar set is complete polar in the above sense, since in this case $p_{m}$ can be chosen to be a Fekete polynomial of $L$ times a constant (see also Proposition 4.1).

The goal of this article is to study a class of pluripolar subsets of $d o-$ mains in $\mathbb{C}^{n}$ which are reminiscent of complete pluripolar sets in the sense of Sadullaev. We refer to them as $S$-complete pluripolar sets. Here is a brief outline of the paper. In Section 2, we recall some elements of pluripotential theory pertaining to our work and introduce the concept of $S$-complete

2000 Mathematics Subject Classification: 32F05, 33C10.

Key words and phrases: pluripolar set, plurisubharmonic function, pseudoconvex domain. 
pluripolar sets. Section 3 contains basic properties of $S$-complete pluripolar sets. Proposition 3.1 tells us that a Borel $S$-complete pluripolar set is pluripolar in the usual sense. Moreover, if the domain $D$ is pseudoconvex and $E$ is closed then $E$ is in fact complete pluripolar, again in the usual sense. It is perhaps a little surprising that complete pluripolarity is quite close to $S$-complete pluripolarity. This fact is exhibited in Theorem 3.3. The last result of the section is Proposition 3.6 which explains the motivation for studying $S$-complete pluripolar sets. Namely we show that every holomorphic function on the complement of a closed $S$-complete pluripolar set in a pseudoconvex domain $D$ can be approximated locally uniformly by meromorphic functions on $D$. In the final section, we present explicit examples of $S$-complete pluripolar sets. The simplest ones are Borel polar sets in one dimension and complex hypersurfaces which are defined by global holomorphic functions. The paper ends up with more complicated examples of graphs of holomorphic functions with closed $S$-complete pluripolar singularities. This result is inspired by some recent work on pluripolar hulls of holomorphic graphs (see [EW1], [EW2], [LNT], [N1]).

Acknowledgments. We would like to thank Professor Nguyen Van Khue for his encouragement during the preparation of this paper. It will be clear that we have profited very much from the fundamental paper [Sa]. This work is supported by the National Research Program in Natural Sciences of Vietnam.

II. Preliminaries. An upper semicontinuous function $u$ on a domain $D$ in $\mathbb{C}^{n}$ is called plurisubharmonic if the restriction of $u$ to the intersection of $D$ with every complex line is subharmonic (we allow the function identically $-\infty$ to be plurisubharmonic). The cone of plurisubharmonic functions (resp. negative plurisubharmonic functions) is denoted by $\mathcal{P S H}(D)$ (resp. $\left.\mathcal{P S H ^ { - }}(D)\right)$.

A subset $E$ of $\mathbb{C}^{n}$ is called pluripolar if for every $a \in A$ we can find a neighbourhood $U_{a}$ of $a$ and $u \in \mathcal{P S H}\left(U_{a}\right)$ such that $u \equiv-\infty$ on $E \cap U_{a}$ and $u \not \equiv-\infty$ on any connected component of $U_{a}$. A basic theorem of Josefson (see [Kl, Theorem 4.7.4]) asserts that if $E$ is pluripolar in $\Omega$ then there exists a plurisubharmonic function $u$ on $\mathbb{C}^{n}$ such that $u \equiv-\infty$ on $E$ but $u \not \equiv-\infty$.

If $E$ is pluripolar and contained in some domain $\Omega$ of $\mathbb{C}^{n}$ then we say that $E$ is complete pluripolar in $D$ if there exists $u \in \mathcal{P S H}(D)$ such that $u^{-1}(-\infty)=E$. Obviously every complete pluripolar set $E \subset \Omega$ is a $G_{\delta}$, it is also well known in one dimension that every $G_{\delta}$ polar set is complete polar. However, the situation changes drastically in higher dimensions. The analytic set $\{(z, 0):|z|<1\}$ is complete pluripolar in the bidisk $\{(z, w)$ : $|z|<1,|w|<1\}$ but not in any neigbourhood of the closed bidisk. 
A useful tool in studying complete pluripolar sets is pluripolar hulls introduced by Levenberg and Poletsky in [LP]. More precisely, for a given pluripolar subset $E$ of a domain $D$ in $\mathbb{C}^{n}$, we define

$$
\begin{aligned}
& E_{D}^{*}=\bigcap\left\{z \in D: u(z)=-\infty, u \in \mathcal{P} \mathcal{S H}(D),\left.u\right|_{E} \equiv-\infty\right\}, \\
& E_{D}^{-}=\bigcap\left\{z \in D: u(z)=-\infty, u \in \mathcal{P S H}^{-}(D),\left.u\right|_{E} \equiv-\infty\right\} .
\end{aligned}
$$

It is trivial that $E \subset E_{D}^{*} \subset E_{D}^{-}$, and if $E$ is complete pluripolar in $D$ then $E_{D}^{*}=E$.

The following result of Levenberg and Poletsky (see [LP]) is very useful when we want to "localize" $E_{D}^{*}$.

Theorem 2.1. Let $D$ be a pseudoconvex domain in $\mathbb{C}^{n}$ and $\left\{D_{j}\right\}_{j \geq 1}$ be an increasing sequence of relatively compact domains with $\bigcup D_{j}=D$. Then for every pluripolar subset $E$ of $D$ we have

$$
E_{D}^{*}=\bigcup_{j \geq 1}\left(E \cap D_{j}\right)_{D_{j}}^{-} .
$$

Moreover, if $D$ is hyperconvex, i.e., there exists a negative plurisubharmonic exhaustion function on $D$, then $E_{D}^{*}=E_{D}^{-}$.

The result below, due to Zeriahi (Proposition 2.1 in [Ze]), characterizes complete pluripolarity of a set $E$ in terms of pluripolar hulls.

TheOREM 2.2. Let $E$ be a pluripolar subset of a pseudoconvex domain $D$ in $\mathbb{C}^{n}$. Then $E$ is complete pluripolar in $D$ if $E_{D}^{*}=E$ and $E$ is an $F_{\sigma}$ and $G_{\delta}$ set.

Now we introduce the following concept which is an adaptation of the concept of complete pluripolar in [Sa].

Definition 2.3. Let $D$ be a domain in $\mathbb{C}^{n}$ and $E$ be a subset of $D$. We say that $E$ is $S$-complete pluripolar in $D$ if for every subdomain $D^{\prime} \subset \subset D$, and any compact sets $K \subset D^{\prime} \backslash E$ and $L \subset D^{\prime} \cap E$, there are positive constants $C, \delta$, a sequence $\left\{p_{m}\right\}_{m \geq 1}$ of holomorphic functions on $D$ and a sequence of positive integers $\left\{a_{m}\right\}_{m \geq 1}$ such that

(a) $\inf _{K} \frac{1}{a_{m}} \log \left|p_{m}\right|>\log \delta$,

(b) $\sup _{L} \frac{1}{a_{m}} \log \left|p_{m}\right|<-\log m$,

(c) $\sup _{D^{\prime}} \frac{1}{a_{m}} \log \left|p_{m}\right|<C$.

Some remarks should be made at this point. First, it is enough to check the conditions (a)-(c) for $D^{\prime}$ running over an exhaustion of $D$ by relatively 
compact subdomains. Second, (a)-(c) need to be satisfied only for sufficiently large $m$. Third, if $E$ is closed in $D$ then (b) can be replaced by

$$
\left(\mathrm{b}^{\prime}\right) \sup _{E \cap D^{\prime}} \frac{1}{a_{m}} \log \left|p_{m}\right|<-\log m \text {. }
$$

Fourth, if $D$ is pseudoconvex then $p_{m}$ has to be holomorphic only on $D^{\prime}$. To see this, just consider a relatively compact subdomain $D^{\prime \prime}$ of $D$ such that $D^{\prime} \subset \subset D^{\prime \prime}$ and $\left(D^{\prime \prime}, D\right)$ is a Runge pair, i.e., every holomorphic function on $D^{\prime \prime}$ can be approximated uniformly on compact subsets by holomorphic functions on $D$. Finally, if $D=\mathbb{C}^{n}$ and $E$ is closed and complete pluripolar in the sense of Sadullaev, then a direct application of Bernstein-Walsh's inequality shows that $E$ is $S$-complete pluripolar. However, we do not know if the converse implication is true.

III. Basics on $S$-complete pluripolar sets. We begin with the following

Proposition 3.1. Every $\mathcal{K}$-analytic and $S$-complete pluripolar subset $E$ in a domain $D$ is pluripolar. Moreover, if $E$ is an $F_{\sigma}$ and $G_{\delta}$ set and $D$ is pseudoconvex then $E$ is complete pluripolar in $D$.

Here a set $E \subset D$ is called $\mathcal{K}$-analytic if it may be obtained by a Suslin operation on compact subsets of $D$. In particular, every Borel subset of $D$ is $\mathcal{K}$-analytic.

The following simple fact is needed in the proof.

Lemma 3.2. Let $\Omega$ be a pseudoconvex domain in $\mathbb{C}^{n}$ and $E_{j}, j=1,2, \ldots$, be pluripolar subsets of $\Omega$. Set $E=\bigcup_{j=1}^{\infty} E_{j}$. Then

$$
E_{\Omega}^{*}=\bigcup_{j=1}^{\infty}\left(E_{j}\right)_{\Omega}^{*} .
$$

In particular, if $E_{j}$ is complete pluripolar in $\Omega$ for each $j \geq 1$ and $E$ is an $F_{\sigma}$ and $G_{\delta}$ set then $E$ is complete pluripolar.

Proof. We first assume that $\Omega$ is bounded hyperconvex, i.e., there exists a negative continuous plurisubharmonic exhaustion function for $\Omega$. It is clear that $\bigcup_{j=1}^{\infty}\left(E_{j}\right)_{\Omega}^{*} \subset E_{\Omega}^{*}$. Pick $z_{0} \notin \bigcup_{j=1}^{\infty}\left(E_{j}\right)_{\Omega}^{*}$. Since $\Omega$ is hyperconvex, it follows from Theorem 2.1 that $\left(E_{j}\right)_{\Omega}^{*}=\left(E_{j}\right)_{\Omega}^{-}$for all $j \geq 1$, so there exists $u_{j} \in \mathcal{P S H}^{-}(\Omega)$ such that $u_{j} \equiv-\infty$ on $E_{j}$ and $u_{j}\left(z_{0}\right)>-\infty$. By multiplying $u_{j}$ with a suitable positive constant we can achieve that $u_{j}\left(z_{0}\right)>-2^{-j}$. Set $u(z)=\sum_{j=1}^{\infty} u_{j}(z)$. Then $u<0, u \in \mathcal{P S H}^{-}(\Omega), u\left(z_{0}\right)>-1$, and $\left.u\right|_{E} \equiv-\infty$. This implies that $z_{0} \notin E_{\Omega}^{*}$. Thus the equality (1) holds in case $\Omega$ is hyperconvex. 
Next, suppose that $\Omega$ is an arbitrary pseudoconvex domain. Let $\left\{\Omega_{k}\right\}$ be an increasing sequence of bounded hyperconvex domains with $\Omega_{k} \subset \subset \Omega_{k+1}$ and $\bigcup_{j=1}^{\infty} \Omega_{k}=\Omega$. In view of Theorem 2.1 and the above we have

$$
\begin{aligned}
E_{\Omega}^{*} & =\bigcup_{k=1}^{\infty}\left(E \cap \Omega_{k}\right)_{\Omega_{k}}^{-}=\bigcup_{k=1}^{\infty}\left(\left(\bigcup_{j=1}^{\infty} E_{j}\right) \cap \Omega_{k}\right)_{\Omega_{k}}^{-}=\bigcup_{k=1}^{\infty}\left(\bigcup_{j=1}^{\infty}\left(E_{j} \cap \Omega_{k}\right)\right)_{\Omega_{k}}^{-} \\
& =\bigcup_{k=1}^{\infty} \bigcup_{j=1}^{\infty}\left(E_{j} \cap \Omega_{k}\right)_{\Omega_{k}}^{-}=\bigcup_{j=1}^{\infty} \bigcup_{k=1}^{\infty}\left(E_{j} \cap \Omega_{k}\right)_{\Omega_{k}}^{-}=\bigcup_{j=1}^{\infty}\left(E_{j}\right)_{\Omega^{*}}^{*} .
\end{aligned}
$$

If the $E_{j}$ are complete pluripolar then $E=E_{\Omega}^{*}$, and applying Theorem 2.2 we deduce that $E$ is complete pluripolar in $\Omega$.

Proof of Proposition 3.1. Fix a domain $D^{\prime} \subset \subset D$, a compact $L \subset D^{\prime} \cap E$ and an arbitrary point $z_{0}$ in $D^{\prime} \backslash E$. Then there exist $C, \delta>0$ such that for each $m \geq 1$, there exist a holomorphic function $p_{m}$ on $D$ and $a_{m} \geq 1$ satisfying

$\frac{1}{a_{m}} \log \left|p_{m}\left(z_{0}\right)\right|>\log \delta, \quad \sup _{L} \frac{1}{a_{m}} \log \left|p_{m}\right| \leq-\log m, \quad \sup _{D^{\prime}} \frac{1}{a_{m}} \log \left|p_{m}\right|<C$. Take a sequence of positive numbers $b_{m}$ such that $\sum_{m=1}^{\infty} b_{m}<\infty$ and $\sum_{m=1}^{\infty} b_{m} \log m=\infty$. Set

$$
u(z)=\sum_{m \geq 2} b_{m}\left(\frac{1}{a_{m}} \log \left|p_{m}(z)\right|-C\right) .
$$

It is clear that $u$ is the decreasing limit of a sequence of negative plurisubharmonic functions on $D^{\prime}$. Moreover, $u\left(z_{0}\right)>-\infty$ and $u \equiv-\infty$ on $L$. This implies that $L$ is pluripolar for every compact $L \subset E \cap D^{\prime}$, hence by Theorem 8.3 in $[\mathrm{BT}], E \cap D^{\prime}$ is pluripolar and then so is $E$.

Now, assume that $D$ is pseudoconvex. Let $\left\{D_{j}\right\}$ be an increasing sequence of bounded hyperconvex subdomains of $D$ such that $D_{j} \subset \subset D_{j+1}$ and $\bigcup_{j \geq 1} D_{j}=D$. Since $E$ is $F_{\sigma}$, take a sequence $\left\{L_{k}\right\}$ of compact subset of $E$ such that $\bigcup_{k \geq 1} L_{k}=E$. It follows from the above proof that $\bigcup_{k=1}^{\infty}\left(L_{k} \cap D_{j}\right)_{D_{j}}^{-}=E \cap D_{j}$. Applying Lemma 3.2 we get

$$
\left(E \cap D_{j}\right)_{D_{j}}^{-}=\bigcup_{k \geq 1}\left(L_{k} \cap D_{j}\right)_{D_{j}}^{-}=E \cap D_{j}
$$

Combining this with Theorem 2.1 one obtains

$$
E_{D}^{*}=\bigcup_{j \geq 1}\left(E \cap D_{j}\right)_{D_{j}}^{-}=E .
$$

Now the conclusion follows from Theorem 2.2.

The main result of this section is the following theorem which establishes connections between $S$-complete pluripolarity and complete pluripolarity in the usual sense. 
Theorem 3.3. Let $D$ be a pseudoconvex domain in $\mathbb{C}^{n}$ and $E$ be closed subset of D. Then:

(a) If $E$ is $S$-complete pluripolar in $D$ then $D \backslash E$ is pseudoconvex and $E$ is complete pluripolar.

(b) If $E$ is complete pluripolar in $D$ then for every relatively compact subdomain $D^{\prime} \subset D$, any compact set $K \subset D^{\prime} \backslash E$ with $\lambda(K)>0$ ( $\lambda$ denotes the Lebesgue measure in $\mathbb{C}^{n}$ ), every finite set $A \subset K$ and every $\varepsilon>0$ there exist constants $C>0,0<\delta<1$, a compact $K^{\prime}$, a sequence $\left\{p_{m}\right\}$ of holomorphic functions on $D$ and a sequence of positive integers $a_{m} \geq 1$ that satisfy

(i) $A \subset K^{\prime} \subset K, \lambda\left(K^{\prime}\right)>\lambda(K)-\varepsilon$,

(ii) $\inf _{K^{\prime}} \frac{1}{a_{m}} \log \left|p_{m}\right|>\log \delta, \sup _{D^{\prime} \cap E} \frac{1}{a_{m}} \log \left|p_{m}\right| \leq-\log m$,

$$
\sup _{D^{\prime}} \frac{1}{a_{m}} \log \left|p_{m}\right|<C .
$$

Proof. (a) Since a closed subset of $D$ is an $F_{\sigma}$ and $G_{\delta}$ set, Proposition 3.2 shows that $E$ is complete pluripolar in $D$. Fix a pseudoconvex relatively compact domain $D^{\prime}$ in $D$. It suffices to show that $D^{\prime} \backslash E$ is pseudoconvex. Let $\left\{K_{m}\right\}$ and $\left\{L_{m}\right\}$ be increasing sequences of compact subsets of $D^{\prime} \backslash E$ and $D^{\prime} \cap E$ respectively such that $\bigcup K_{m}=D^{\prime} \backslash E$ and $\bigcup L_{m}=D^{\prime} \cap E$. It follows from $S$-complete pluripolarity of $E$ that there are sequences $\left\{\delta_{m}\right\}$, $\left\{p_{m}\right\},\left\{a_{m}\right\}$ where $\delta_{m} \downarrow 0, a_{m} \geq 1$ and $p_{m}$ is holomorphic on $D$ such that

$$
\inf _{K_{m}} \frac{1}{a_{m}} \log \left|p_{m}\right|>\log \delta_{m}, \quad \sup _{L_{m}} \frac{1}{a_{m}} \log \left|p_{m}\right|<\log \left(\delta_{m} / 2\right) .
$$

Set

$$
D_{m}^{\prime}=\left\{z \in D: \frac{1}{a_{m}} \log \left|p_{m}(z)\right|>\log \delta_{m}\right\} .
$$

As $D$ is pseudoconvex and $p_{m}$ is holomorphic on $D$ it follows that $D_{m}^{\prime}$ is an open pseudoconvex set. Oka's theorem (see [Hö]) shows that the function $-\log d\left(z, \partial D_{m}^{\prime}\right)$ is plurisubharmonic on $D_{m}^{\prime}$, where $d(z, \partial \Omega)$ denotes the Euclidean distance from $z$ to $\partial \Omega$. Now we infer from (2) that $-\log d\left(z, \partial D_{m}^{\prime}\right)$ converges pointwise to $-\log d(z, \partial(D \backslash E))$. Thus, Oka's theorem implies that $D \backslash E$ is pseudoconvex, completing the proof.

(b) Since $E$ is closed complete pluripolar in $D$, according to Lemma 2.1 in [Ze] (see also Lemma 4.2 in [EW2] and Proposition 3.1 in [LNT]), we can find $\varphi \in \mathcal{P S H}(D)$ such that $e^{\varphi}$ is continuous on $D$ and $\varphi=-\infty$ precisely on $E$. Using the approximation theorem of Fornæss and Narasimhan (see Theorem 5.5 in $[\mathrm{FN}]$ ) we get a sequence $\left\{\varphi_{m}\right\}$ of $\mathcal{C}^{\infty}$ smooth strictly 
plurisubharmonic functions on $D$ such that $\varphi_{m} \downarrow \varphi$ on $D$. Set

$$
C=\sup _{D^{\prime}} \varphi+1, \quad \alpha=\inf _{K} \varphi-1>-\infty .
$$

By passing to a subsequence we may achieve that $\varphi_{m} \leq-\log (m+1)$ on $D^{\prime} \cap E$ and $\varphi_{m}<C-1 / 2$ on $K$ for all $m \geq 1$. Next by the proof of Theorem 4.2.13 in [Hö] we get a sequence $\left\{p_{j, m}\right\}$ of holomorphic functions on $D$ and a sequence $\left\{a_{j, m}\right\}$ of positive integers such that the sequence $\left(1 / a_{j, m}\right) \log \left|p_{j, m}\right|$ is locally uniformly upper bounded, $\left(1 / a_{j, m}\right) \log \left|p_{j, m}\right| \rightarrow \varphi_{m}$ in $L_{\mathrm{loc}}^{1}(D)$ and $\left(1 / a_{j, m}\right) \log \left|p_{j, m}(z)\right| \rightarrow \varphi_{m}(z)$ for every $z \in A$. It follows that

$$
\limsup _{j \rightarrow \infty} \frac{1}{a_{j, m}} \log \left|p_{j, m}(z)\right|=\varphi_{m}(z), \quad \forall z \in \mathbb{C}^{n} .
$$

Using Hartogs' lemma, for every $m \geq 1$ there is $j_{m}$ so large that

$$
\begin{gathered}
\left\|p_{j_{m}, m}\right\|_{D^{\prime} \cap E} \leq(1 / m)^{a_{j_{m}, m}}, \quad\left\|p_{j_{m}, m}\right\|_{D^{\prime}}<C^{a_{m}}, \\
\left|p_{j_{m}, m}(z)\right|>e^{-a_{j_{m}, m}(|\alpha|+1)}, \quad \forall z \in A,
\end{gathered}
$$

and

$$
\int_{K}\left|\frac{1}{a_{j_{m}, m}} \log \right| p_{j_{m}, m}|-\varphi| d \lambda<\frac{1}{m^{2}} .
$$

For $m \geq 1$ we set

$$
A_{m}=\left\{z \in K: \frac{1}{a_{j_{m}, m}} \log \left|p_{j_{m}, m}(z)\right|<-|\alpha|-2 / \varepsilon\right\} .
$$

It follows that $\lambda\left(A_{m}\right)<\varepsilon / 2 m^{2}$ for all $m \geq 1$. Thus we have

$$
\lambda\left(\bigcup_{m \geq 1} A_{m}\right) \leq \sum_{m \geq 1} \lambda\left(A_{m}\right)<\varepsilon .
$$

Set $K^{\prime}:=K \backslash \bigcup A_{m}$. Then $\lambda\left(K^{\prime}\right)>\lambda(K)-\varepsilon$. Obviously we also have

$$
\frac{1}{a_{j_{m}, m}} \log \left|p_{j_{m}, m}(z)\right| \geq-|\alpha|-2 / \varepsilon, \quad \forall m \geq 1, \forall z \in K^{\prime} .
$$

The proof of this part is accomplished by setting

$$
\delta:=e^{-|\alpha|-2 / \varepsilon}, \quad a_{m}:=a_{j_{m}, m}, \quad p_{m}:=p_{j_{m}, m} .
$$

To finish this section we make some conjectures.

Conjecture 3.4. If $E$ is a complex hypersurface of a pseudoconvex domain $D$ then $E$ is $S$-complete pluripolar in $D$.

Conjecture 3.5. If $E$ is a closed complete pluripolar subset of a pseudoconvex domain $D$ and if $D \backslash E$ is pseudoconvex then $E$ is $S$-complete pluripolar in $D$. 
According to Proposition 9.1 in [Sk], every complex subvariety of a pseudoconvex domain $D$ in $\mathbb{C}^{n}$ is the common zero set of $n+1$ holomorphic functions on $D$, in particular it is complete pluripolar in $D$. Thus Conjecture 3.5 implies Conjecture 3.4.

The interest in $S$-complete pluripolar sets stems from the following approximation result, which is implicitly contained in Section 3 of [Sa]. For an analogous result for complete pluripolar sets see Proposition 3.2 in [N2].

Proposition 3.6. Let $E$ be a closed $S$-complete pluripolar subset of a pseudoconvex domain $D$ in $\mathbb{C}^{n}$ and $f$ be a holomorphic function on $D \backslash E$. Then for every compact $K \subset D \backslash E$ and $\varepsilon>0$ there are holomorphic functions $p, q$ on $D$ such that $\|f-p / q\|_{K}<\varepsilon$.

Proof. We will use a method devised by Chirka and Sadullaev in [Ch] and [Sa]. Pick a relatively compact pseudoconvex domain $D^{\prime}$ of $D$ such that $K \subset D^{\prime}$ and $\left(D^{\prime}, D\right)$ is a Runge pair. Since $E$ is closed $S$-complete pluripolar, there are a sequence $\left\{p_{m}\right\}$ of holomorphic functions on $D$ and a sequence $\left\{a_{m}\right\}$ of positive integers such that

$$
\inf _{K}\left|p_{m}\right|>\delta^{a_{m}}, \quad\left\|p_{m}\right\|_{E \cap D^{\prime}}<(1 / m)^{a_{m}} .
$$

For each $m \geq 1$, consider the pseudoconvex domain $U_{m}=D^{\prime} \times\{w:|w|<$ $\left.m^{a_{m}}\right\}$ with the complex hypersurface $A_{m}=\left\{(z, w) \in U_{m}: p_{m}(z) w=1\right\}$. It follows from (3) that the function $\widehat{f}(z, w)$ equal to $f(z)$ on $A_{m}$ is holomorphic on $A_{m}$. By Cartan's theorem, there exists a holomorphic function $F_{m}$ on $U_{m}$ such that $\left.F_{m}\right|_{A}=\widehat{f}$. Expanding $F$ in Hartogs series we get

$$
F_{m}(z, w)=\sum_{j=0}^{\infty} f_{j, m}(z) w^{j},
$$

where the $f_{j, m}$ are holomorphic functions on $D^{\prime}$, and the series converges locally uniformly on $U_{m}$. Substituting $w=1 / p_{m}(z)$ we obtain

$$
f(z)=\sum_{j=0}^{\infty} \frac{f_{j, m}(z)}{p_{m}^{j}(z)},
$$

where the series converges locally uniformly on $D^{\prime} \cap\left\{z:\left|p_{m}(z)\right|>(1 / m)^{a_{m}}\right\}$. In particular, it converges uniformly on $K$ if $m \geq m_{1}:=[1 / \delta]+1$, where $[x]$ denotes the largest integer not exceeding $x$. Now the conclusion follows as we can approximate $f_{j, m}$ uniformly on $K$ by holomorphic functions on $D$.

IV. Examples of $S$-complete pluripolar sets. We start with the following simple facts:

Proposition 4.1. Let $E$ be a Borel subset of a domain $D$ in $\mathbb{C}^{n}$. Then $E$ is $S$-complete pluripolar in each of the following cases. 
(i) $n=1$ and $E$ is polar in $D$.

(ii) $E$ is the zero set of a holomorphic function $f$ on $D, f \not \equiv 0$.

Proof. (i) This result follows almost immediately from classical facts of potential theory. However, for the reader's convenience we recall some details. Assume that $E$ is a Borel polar set in $\mathbb{C}$ and $D$ is a domain containing $E$. Let $D^{\prime}$ be a relatively compact subdomain of $D$, and let $K \subset D^{\prime} \backslash E$ and $L \subset D^{\prime} \cap E$ be compact. Let $p_{m}$ be a Fekete polynomial for $L$ of degree $m$, i.e., $p_{m}(z)=\prod_{j=1}^{m}\left(z-w_{i}\right)$, where $\left\{w_{1}, \ldots, w_{m}\right\}$ is a Fekete $m$-tuple for $L$ (see Definition 5.5.3 in [Ra]). Then by Theorem 5.5.4 in [Ra],

$$
\sup _{L} \frac{1}{m} \log \left|p_{m}\right| \leq \log \left(\delta_{m}(L)\right),
$$

where $\delta_{m}(L)$ is the $m$ th diameter of $L$ (see Definition 5.5.1 in [Ra]). Now, by Theorem 5.5.2 in [Ra] we have $\lim _{m \rightarrow \infty} \delta_{m}(L)=0$. Thus, $\sup _{L} m^{-1} \log \left|p_{m}\right|$ $\leq-\log m$ for $m$ sufficiently large. Moreover, for $z \in K$ we have

$$
\frac{1}{m} \log \left|p_{m}(z)\right|=\frac{1}{m} \sum_{j=1}^{m} \log \left|z-w_{j}\right| \geq \log \delta
$$

where $\delta=\operatorname{dist}(K, L)>0$. This implies that $\inf _{K} m^{-1} \log \left|p_{m}\right| \geq \log \delta$. Finally, on $D^{\prime}$ we have $m^{-1} \log \left|p_{m}(z)\right| \leq \log \left(\delta_{1}\left(D^{\prime}\right)\right)$. Thus $E$ is $S$-complete polar in $D$.

(ii) Let $D^{\prime}$ be a relatively compact subdomain of $D$. If $K$ and $L$ are compact subsets of $D^{\prime} \backslash E$ and $D^{\prime} \cap E$ respectively, then for $m \geq 1$ we choose $C, \delta, p_{m}, a_{m}$ such that

$$
\log C=\sup _{D^{\prime}} \log |f|+1, \quad \log \delta=\inf _{K} \log |f|-1, \quad a_{m}=1, \quad p_{m}=f .
$$

It is clear that these choices satisfy (a)-(c) of Definition 2.3. Thus $E$ is $S$-complete pluripolar in $D$.

Remark. Proposition 4.1(i) is not true when $n \geq 2$. Indeed, from Theorem 3.3 and the Hartogs extension theorem we infer that no compact pluripolar subset of a pseudoconvex domain $D \subset \mathbb{C}^{n}$ is $S$-complete pluripolar in $D$.

It is easy to see that if $f: D_{1} \rightarrow D_{2}$ is an open holomorphic mapping between domains in $\mathbb{C}^{n}$ and $\mathbb{C}^{m}$ then $f^{-1}(E)$ is $S$-complete pluripolar in $D_{1}$ for every $S$-complete pluripolar subset $E$ in $D_{2}$. The following result shows that $S$-complete pluripolarity is invariant under proper holomorphic transformations.

Proposition 4.2. Let $f: D_{1} \rightarrow D_{2}$ be a proper holomorphic mapping between domains in $\mathbb{C}^{n}$ and let $E$ be an $S$-complete pluripolar subset in $D_{1}$. Then $f(E)$ is $S$-complete pluripolar in $D_{2}$. 
Proof. Since $f$ is proper, there is $k \geq 1$ and a complex subvariety $V$ (possibly empty) of $D_{1}$ such that $f$ is a local biholomorphism on $D_{1} \backslash V$ and $f^{-1}(z)$ consists of exactly $k$ distinct points for $z \in D_{2} \backslash f(V)$. Let $D^{\prime}$ be a relatively compact subdomain of $D_{2}$, and let $K$ and $L$ be compact subsets of $D^{\prime} \backslash f(E)$ and $f(E) \cap D^{\prime}$ respectively. We have to show that there are positive constants $C, \delta$ and a sequence $\left\{p_{m}\right\}$ of holomorphic functions satisfying the conditions (a)-(c) of Definition 2.3. Since $f(V)$ is a complex subvariety of $D_{2}$, enlarging $K$ we may assume that $f(V)$ is nowhere dense in $K$. Now we apply the $S$-complete pluripolarity of $E$ in $D_{1}$ to the open set $D^{\prime \prime}=f^{-1}\left(D^{\prime}\right)$ and the compact sets $K^{\prime}:=f^{-1}(K), L^{\prime}:=f^{-1}(L)$ to obtain a sequence $\left\{q_{m}\right\}$ of holomorphic functions on $D_{1}$ and positive constants $C, \delta$ such that

$$
\begin{array}{ll}
\frac{1}{a_{m}} \log \left|q_{m}\right|>\log \delta & \text { on } K^{\prime}, \\
\frac{1}{a_{m}} \log \left|q_{m}\right|<C & \text { on } D^{\prime \prime}, \\
\frac{1}{a_{m}} \log \left|q_{m}\right|<-\log m & \text { on } L^{\prime} .
\end{array}
$$

Now we define on $D_{2} \backslash f(V)$ the function

$$
p_{m}(z)=\prod_{f(\xi)=z} q_{m}(\xi) .
$$

It is holomorphic, since it is locally a product of $k$ holomorphic functions. Observe that $p_{m}$ is locally bounded near every point of the complex subvariety $f(V)$, so by Riemann's extension theorem we can extend $p_{m}$ to a holomorphic function, still denoted by $p_{m}$, on $D_{2}$. It follows from (4)-(6) that

$$
\begin{aligned}
& \frac{1}{k a_{m}} \log \left|p_{m}\right|>\log \delta \quad \text { on } K \backslash f(V), \\
& \frac{1}{k a_{m}} \log \left|p_{m}\right|<C \quad \text { on } D^{\prime} \backslash f(V) \text {, } \\
& \frac{1}{k a_{m}} \log \left|p_{m}\right|<-\log m \quad \text { on } L \backslash f(V) \text {. }
\end{aligned}
$$

As $f(V)$ is nowhere dense in $K$, we infer that the inequalities in (7) and (8) hold throughout $K$ and $D^{\prime}$ respectively. It remains to show that (9) is also true on $L$. For this we let $\left\{V_{j}\right\}$ be a sequence of open subsets of $D_{2}$ decreasing to $L$. Choose $\varepsilon>0$ so small that $\left(1 / a_{m}\right) \log \left|q_{m}\right|<-\log m-\varepsilon$ on $L^{\prime}$. Since $f$ is proper, in view of (4) we can choose $j(\varepsilon)$ so large that

$$
\frac{1}{a_{m}} \log \left|q_{m}\right|<-\log m-\varepsilon / 2 \quad \text { on } f^{-1}\left(V_{j(\varepsilon)}\right) .
$$


This implies that

$$
\frac{1}{k a_{m}} \log \left|p_{m}\right|<-\log m-\varepsilon / 2 \quad \text { on } V_{j(\varepsilon)} \backslash f(V) .
$$

Hence this inequality holds on $V_{j(\varepsilon)}$, in particular on $L$. We are done.

The next result should be compared to Proposition 2 in [Sa].

Proposition 4.3. A countable union of closed S-complete pluripolar sets in a domain $D$ is $S$-complete pluripolar in $D$.

Proof. The proof proceeds in two steps.

STEP 1. We prove that the union of two closed $S$-complete pluripolar sets $E_{1}, E_{2}$ is also $S$-complete pluripolar. Let $D^{\prime} \subset \subset D$, and let $K \subset D^{\prime} \backslash E$ and $L \subset E \cap D^{\prime}$ be compact sets. Since $E_{1}, E_{2}$ are closed, $L \cap E_{1}$ and $L \cap E_{2}$ are compact in $E_{1} \cap D^{\prime}$ and $E_{2} \cap D^{\prime}$ respectively. Since $E_{1}$ and $E_{2}$ are $S$-complete pluripolar, there are constants $C_{1}, C_{2}, \delta_{1}, \delta_{2}>0$ such that for every $m \geq 1$ there are sequences $\left\{p_{1, m}\right\},\left\{p_{2, m}\right\},\left\{a_{m}\right\},\left\{b_{m}\right\}$ where $p_{m}, q_{m}$ are holomorphic on $D$ and $a_{m}, b_{m}$ are positive integers satisfying

$$
\begin{array}{cc}
\inf _{K} \frac{1}{a_{m}} \log \left|p_{1, m}\right|>\log \delta_{1}, & \inf _{K} \frac{1}{b_{m}} \log \left|p_{2, m}\right|>\log \delta_{2}, \\
\sup _{L \cap E_{1}} \frac{1}{a_{m}} \log \left|p_{1, m}\right|<-\log m, & \sup _{L \cap E_{2}} \frac{1}{b_{m}} \log \left|p_{2, m}\right|<-\log m, \\
\sup _{D^{\prime}} \frac{1}{a_{m}} \log \left|p_{1, m}\right|<C_{1}, & \sup _{D^{\prime}} \frac{1}{b_{m}} \log \left|p_{2, m}\right|<C_{2} .
\end{array}
$$

Set $C=C_{1}+C_{2}, \delta=\delta_{1} \delta_{2}$ and $p_{m}(z)=\left(p_{1, m}(z)\right)^{b_{m}}\left(p_{2, m}(z)\right)^{a_{m}}$ for $z \in D$. Then it follows from (10)-(12) that

$$
\begin{aligned}
& \inf _{K} \frac{1}{a_{m} b_{m}} \log \left|p_{m}\right|>\log \delta, \quad \sup _{L} \frac{1}{a_{m} b_{m}} \log \left|p_{m}\right|<-\log m, \\
& \sup _{D^{\prime}} \frac{1}{a_{m} b_{m}} \log \left|p_{m}\right|<C .
\end{aligned}
$$

This implies that $E$ is $S$-complete pluripolar.

STEP 2. We move to the general case. Let $D^{\prime} \subset \subset D$, and let $K \subset D^{\prime} \backslash E$ and $L \subset E \cap D^{\prime}$ be compact sets. By Step 1 , after replacing $E_{k}$ by $\bigcup_{j=1}^{k} E_{j}$, we can assume that $E_{k}$ is an increasing sequence. Since $E_{k}$ is $S$-complete pluripolar, there are $C_{k}>\log k, 0<\delta_{k}<k$, a sequence $\left\{p_{k, m}\right\}_{m \geq 1}$ of holomorphic functions on $D$ and a sequence $\left\{a_{k, m}\right\}_{m \geq 1}$ of positive integers such that

$$
\begin{aligned}
& \inf _{K} \frac{1}{a_{k, m}} \log \left|p_{k, m}\right|>\log \delta_{k}, \quad \sup _{L \cap E_{k}} \frac{1}{a_{k, m}} \log \left|p_{k, m}\right|<-\log m, \\
& \sup _{D^{\prime}} \frac{1}{a_{k, m}} \log \left|p_{k, m}\right|<C_{k} .
\end{aligned}
$$


Choose a sequence $\left\{b_{k, m}\right\}$ of positive numbers such that

$$
\sum_{m=1}^{\infty} b_{k, m}=\alpha_{k}<\infty, \quad \sum_{m=1}^{\infty} b_{k, m} \log m=\infty, \quad \sum_{k=1}^{\infty} \alpha_{k}\left(C_{k}-\log \delta_{k}\right)<\infty .
$$

For instance, we can take

$$
b_{k, m}:=\frac{1}{2^{k}\left(C_{k}-\log \delta_{k}\right) m(\log m)^{2}} .
$$

Perturbing $b_{k, m}$ slightly, we can achieve that the $b_{k, m}$ are positive rational numbers. For each $k \geq 1$, consider the function

$$
u_{k}(z)=\sum_{m=1}^{\infty} b_{k, m}\left(\frac{1}{a_{k, m}} \log \left|p_{k, m}(z)\right|-C_{k}\right) .
$$

Then $u_{k} \in \mathcal{P} \mathcal{S H}^{-}\left(D^{\prime}\right), u_{k} \equiv-\infty$ on $L \cap E_{k}$ and

$$
u_{k}(z) \geq \sum_{m=1}^{\infty} b_{k, m}\left(\log \delta_{k}-C_{k}\right)=\alpha_{k}\left(\log \delta_{k}-C_{k}\right), \quad \forall z \in K .
$$

Observe that for $z_{0} \in L$ we have $\sum_{k \geq 1} u_{k}\left(z_{0}\right)=-\infty$, so for every $l \geq 1$ there exists $k_{l}$ sufficiently large such that $\sum_{k=1}^{k_{l}} u_{k}\left(z_{0}\right)<-\log l$. It follows that

$$
\sum_{m=1}^{\infty}\left(\sum_{k=1}^{k_{l}} b_{k, m}\left(\frac{1}{a_{k, m}} \log \left|p_{k, m}\left(z_{0}\right)\right|-C_{k}\right)\right)<-\log l .
$$

So there exists $m_{l}$ large enough such that

$$
\sum_{m=1}^{m_{l}}\left(\sum_{k=1}^{k_{l}} b_{k, m}\left(\frac{1}{a_{k, m}} \log \left|p_{k, m}\left(z_{0}\right)\right|-C_{k}\right)\right)<-\log l .
$$

Thus there is an open neighbourhood $G$ of $z_{0}$ in $D$ such that (13) holds for all $z \in G \cap L$. Since $L$ is compact, we may cover it with a finite number of such neighbourhoods to conclude that there are two numbers, which we also denote by $k_{l}$ and $m_{l}$, such that (13) holds for all $z \in L$. Finally, we write $b_{k, m}=r_{k, m} / s_{k, m}$, where $r_{k, m}$ and $s_{k, m}$ are positive integers, and define for $l \geq 1$ the following function on $D$ :

$$
p_{l}(z)=\prod_{k=1}^{k_{l}} \prod_{m=1}^{m_{l}} \frac{\left(p_{k, m}(z)\right)^{M_{l} b_{k, m} / a_{k, m}}}{e^{M_{l} C_{k} b_{k, m}}}
$$

where

$$
M_{l}=\prod_{\substack{1 \leq m \leq m_{l} \\ 1 \leq k \leq k_{l}}} s_{k, m} a_{k, m}
$$


It is clear that $p_{l}$ is holomorphic on $D$; further we deduce from (13) that

$$
\sup _{D^{\prime}} \frac{1}{M_{l}} \log \left|p_{l}\right| \leq 0, \quad \sup _{L} \frac{1}{M_{l}} \log \left|p_{l}(z)\right| \leq-\log l .
$$

On the other hand, for $z \in K$ one has

$$
\frac{1}{M_{l}} \log \left|p_{l}(z)\right|>\sum_{k=1}^{\infty} \alpha_{k}\left(\log \delta_{k}-C_{k}\right)>-\infty .
$$

The proof is completed by taking $\log \delta=\sum_{k=1}^{\infty} \alpha_{k}\left(\log \delta_{k}-C_{k}\right)$.

As a simple consequence of the above result we see that a countable union of complex hypersurfaces which are defined by global holomorphic functions is $S$-complete pluripolar. The following class of $S$-complete pluripolar sets is a little more sophisticated.

Theorem 4.4. Let $D$ be a domain in $\mathbb{C}^{n}$ and $E$ be a closed subset of $D$. Assume that $f$ is a holomorphic function on $D \backslash E$. Let $\Gamma_{f}$ denote the graph of $f$ over $D \backslash E$, i.e.,

$$
\Gamma_{f}:=\{(z, w): z \in D \backslash E, w=f(z)\} .
$$

Then $\Gamma_{f} \cup(E \times \mathbb{C})$ is $S$-complete pluripolar in $D \times \mathbb{C}$ if and only if $E$ is $S$-complete pluripolar in $D$.

We showed in Theorem 3.1 of [N1] an analogous result which says that if $E$ is complete pluripolar in $D$ then $\Gamma_{f} \cup(E \times \mathbb{C})$ is complete pluripolar in $D \times \mathbb{C}$.

Proof. We use the same ideas as in the proof of Theorem 3.1 in [N1]. First we assume that $\Gamma_{f} \cup(E \times \mathbb{C})$ is $S$-complete pluripolar in $D \times \mathbb{C}$. Let $D^{\prime}$ be a relatively compact subdomain of $D$, and let $K$ and $L$ be compact subsets of $D^{\prime} \backslash E$ and $E \cap D^{\prime}$ respectively. Then $f(K)$ is compact in $\mathbb{C}$. Pick $w_{0} \in \mathbb{C} \backslash f(K)$ and a small neighbourhood $U$ of $w_{0}$ in $\mathbb{C} \backslash f(K)$. Using the $S$-complete pluripolarity of $\Gamma_{f} \cup(E \times \mathbb{C})$, for $D^{\prime} \times U, K \times\left\{w_{0}\right\}$ and $L \times\left\{w_{0}\right\}$ we can find $C, \delta>0$, a sequence of holomorphic functions $q_{m}(z, w)$ in $D \times \mathbb{C}$ and a sequence $\left\{a_{m}\right\}$ of positive integers such that

$$
\begin{array}{ll}
\frac{1}{a_{m}} \log \left|q_{m}\right|>\log \delta & \text { on } K \times\left\{w_{0}\right\}, \\
\frac{1}{a_{m}} \log \left|q_{m}\right|<-\log m & \text { on } L \times\left\{w_{0}\right\}, \\
\frac{1}{a_{m}} \log \left|q_{m}\right|<C & \text { on } D^{\prime} \times\left\{w_{0}\right\} .
\end{array}
$$

Let $p_{m}(z)=q_{m}\left(z, w_{0}\right)$ for $z \in D$. Then $\left\{p_{m}\right\}$ satisfies all the required conditions on $D^{\prime}, K$ and $L$. Hence $E$ is $S$-complete pluripolar in $D$. 
For the converse, take pseudoconvex relatively compact subdomains $D^{\prime} \subset \subset D^{\prime \prime} \subset \subset D$, a disk $U \subset \mathbb{C}$ of radius $R$ centred at 0 , and compact sets $K, L$ such that

$$
K \subset\left(D^{\prime} \times U\right) \backslash\left(\Gamma_{f} \cup(E \times U), \quad L \subset\left(\Gamma_{f} \cup(E \times \mathbb{C})\right) \cap\left(D^{\prime} \times U\right) .\right.
$$

Write $L=L_{1} \cup L_{2}$ where $L_{1}=L \cap \Gamma_{f} \cap\left(D^{\prime} \times U\right)$ and $L_{2}=(E \times \mathbb{C}) \cap\left(D^{\prime} \times U\right)$. Then $L_{1} \cap L_{2}=\emptyset$ and $L_{1} \cup L_{2}=L$. Let $\pi: \mathbb{C}^{n+1} \rightarrow \mathbb{C}^{n}, \pi(z, w)=z$. Then $\pi\left(L_{2}\right) \subset E \cap D^{\prime}$ is compact. Since $E$ is $S$-complete pluripolar in $D$, there are constants $C_{1}, \delta_{1}>0$ and two sequences $\left\{q_{m}\right\},\left\{b_{m}\right\}$ where $q_{m}$ is holomorphic on $D$ and $b_{m}$ is a positive integer such that

$$
\begin{aligned}
& \inf _{\pi(K)} \frac{1}{b_{m}} \log \left|q_{m}\right|>\log \delta_{1}, \\
& \sup _{D^{\prime} \cap E} \frac{1}{b_{m}} \log \left|q_{m}\right|<-\log m, \\
& \sup _{D^{\prime}} \frac{1}{b_{m}} \log \left|q_{m}\right|<C_{1} .
\end{aligned}
$$

As in the proof of Proposition 3.6, we set

$$
U_{m}:=D^{\prime \prime} \times\left\{w:|w|<m^{b_{m}}\right\}, \quad A_{m}:=\left\{(z, w) \in U_{m}: p_{m}(z) w=1\right\} .
$$

Then $U_{m}$ is pseudoconvex and $A_{m}$ is a complex hypersurface of $U_{m}$. By an argument similar to the one given in the proof of Proposition 3.6, we may expand

$$
f(z)=\sum_{j=0}^{\infty} \frac{f_{j, m}(z)}{q_{m}^{j}(z)} .
$$

where $f_{j, m}$ are holomorphic functions on $D^{\prime \prime}$ and the series converges locally uniformly on $D^{\prime \prime} \cap\left\{z:\left|q_{m}(z)\right|>(1 / m)^{b_{m}}\right\}$. In particular, it converges uniformly on $\pi(K)$ if $m \geq m_{1}:=[1 / \delta]+1$. Moreover, applying Cauchy's inequalities we also get for each $m$ the following estimate:

$$
\left\|f_{j, m}\right\|_{D^{\prime}} \leq \alpha_{m}\left(\frac{2}{m^{b_{m}}}\right)^{j}, \quad \forall j \geq 0,
$$

where $\alpha_{m}$ is some positive constant independent of $j$. Now for each $m \geq m_{1}$, we define on $D^{\prime} \times \mathbb{C}$ the holomorphic function

$$
p_{m}(z, w)=\left(w-\sum_{j=0}^{\lambda_{m}} \frac{f_{j, m}(z)}{q_{m}^{j}(z)}\right) q_{m}^{2 \lambda_{m}}(z),
$$

where $\left\{\lambda_{m}\right\}$ is an increasing sequence satisfying

$$
\begin{gathered}
2 \log \left(2 \alpha_{m}\right)<b_{m} \lambda_{m}, \quad R<2^{\lambda_{m}} \alpha_{m}, \\
4 \alpha_{m}\left(\frac{2}{m^{b_{m}} \delta_{1}^{b_{m}}}\right)^{\lambda_{m}+1}<\inf _{K}|w-f(z)|=: \delta_{2} .
\end{gathered}
$$


If $(z, w) \in K$ then $z \in \pi(K)$ and from (17),(20) and (22) we deduce

$$
\begin{aligned}
\frac{2}{b_{m} \lambda_{m}} \log \left|p_{m}(z, w)\right| & \frac{2}{b_{m} \lambda_{m}} \log \left|(w-f(z))+\sum_{j \geq \lambda_{m}+1} \frac{f_{j, m}(z)}{q_{m}^{j}(z)}\right|+\frac{4 \log \left|q_{m}(z)\right|}{b_{m}} \\
& >\frac{2}{b_{m} \lambda_{m}} \log \left|\delta_{2}-\sum_{j \geq \lambda_{m}+1}\right| \frac{f_{j, m}(z)}{q_{m}^{j}(z)}||+4 \log \delta_{1} \\
& \geq \frac{2}{b_{m} \lambda_{m}} \log \left|\delta_{2}-\alpha_{m} \sum_{j \geq \lambda_{m}+1}\left(\frac{2}{m^{b_{m}} \delta_{1}^{b_{m}}}\right)^{j}\right|+4 \log \delta_{1} \\
& \geq \frac{2}{b_{m} \lambda_{m}} \log \left|\delta_{2}-2 \alpha_{m}\left(\frac{2}{m^{b_{m}} \delta_{1}^{b_{m}}}\right)^{\lambda_{m}+1}\right|+4 \log \delta_{1} \\
& \geq \frac{2}{b_{m} \lambda_{m}} \log \left(\frac{\delta_{2}}{2}\right)+4 \log \delta_{1}>4 \log \delta_{1}-1
\end{aligned}
$$

for every $m$ sufficiently large .

Next we need a uniform upper bound for $\left(2 / b_{m} \lambda_{m}\right) \log \left|p_{m}\right|$ on $D^{\prime} \times U$. For this, let $C_{2}=\max \left(e^{C_{1}}, 4\right)$. We deduce from (19) that $\left\|q_{m}\right\|_{D^{\prime}} \leq C_{2}^{b_{m}}$. For $(z, w) \in D^{\prime} \times U$, using (20) we obtain

$$
\begin{aligned}
\frac{2}{b_{m} \lambda_{m}} \log \left|p_{m}(z, w)\right| & \leq \frac{2}{b_{m} \lambda_{m}} \log \left(\left|w q_{m}^{2 \lambda_{m}}(z)\right|+\sum_{j=0}^{\lambda_{m}}\left|f_{j, m}(z) q_{m}^{2 \lambda_{m}-j}(z)\right|\right) \\
& \leq \frac{2}{b_{m} \lambda_{m}} \log \left(R C_{2}^{2 b_{m} \lambda_{m}}+\alpha_{m} C_{2}^{2 \lambda_{m} b_{m}} \sum_{j \geq 0}\left(\frac{2}{\left(m C_{2}\right)^{b_{m}}}\right)^{j}\right) \\
& \leq \frac{2}{b_{m} \lambda_{m}} \log \left(R C_{2}^{2 b_{m} \lambda_{m}}+2 \alpha_{m} C_{2}^{2 \lambda_{m} b_{m}}\right)<C,
\end{aligned}
$$

where $C>0$ is some large constant depending only on $C_{2}, R, \delta_{1}$ but not on $m$.

Now we will make some estimates on $L$. If $(z, w) \in L_{2}$ then $z \in \pi\left(L_{2}\right)$, thus by (18),

$$
\frac{1}{b_{m}} \log \left|q_{m}(z)\right|<-\log m \text {. }
$$

So as in the previous estimates we get

$$
\begin{aligned}
\frac{2}{b_{m} \lambda_{m}} \log & \left|p_{m}(z, w)\right| \\
\leq & \frac{2}{b_{m} \lambda_{m}} \log \left(\left|w q_{m}^{\lambda_{m}}(z)\right|+\sum_{j=0}^{\lambda_{m}}\left|f_{j, m}(z) q_{m}^{\lambda_{m}-j}(z)\right|\right)+\frac{2 \log \left|q_{m}(z)\right|}{b_{m}} \\
\leq & =C-2 \log m<-\log m
\end{aligned}
$$

for every $m$ sufficiently large. 
Finally, we deal with $L_{1}$. Split the set $\pi\left(L_{1}\right)$ into two parts,

$$
\begin{aligned}
& L_{m}=\left\{z \in \pi\left(L_{1}\right):\left|q_{m}(z)\right|>4 / m^{b_{m}}\right\}, \\
& L_{m}^{\prime}=\left\{z \in \pi\left(L_{1}\right):\left|q_{m}(z)\right| \leq 4 / m^{b_{m}}\right\} .
\end{aligned}
$$

For $w=f(z)$ with $z \in L_{m}$, we apply (20) and (21) to get

$$
\begin{aligned}
& \frac{2}{b_{m} \lambda_{m}} \log \left|p_{m}(z, f(z))\right| \leq \frac{2}{b_{m} \lambda_{m}} \log \left(\sum_{j \geq \lambda_{m}+1}\left|\frac{f_{j, m}(z)}{q_{m}^{j}(z)}\right|\left|q_{m}(z)\right|^{2 \lambda_{m}}\right) \\
& \quad \leq \frac{2}{b_{m} \lambda_{m}} \log \left(\sum_{j \geq \lambda_{m}+1}\left|\alpha_{m}\left(\frac{2}{m^{b_{m}} q_{m}(z)}\right)^{j}\right|\left|q_{m}(z)\right|^{2 \lambda_{m}}\right) \\
& \quad=\frac{2}{b_{m} \lambda_{m}} \log \left(\alpha_{m}\left(\frac{2}{m^{b_{m}}\left|q_{m}(z)\right|}\right)^{\lambda_{m}+1}\left|q_{m}(z)\right|^{2 \lambda_{m}} \sum_{j \geq 0}\left|\left(\frac{2}{m^{b_{m}} q_{m}(z)}\right)^{j}\right|\right) \\
& \quad=\frac{2}{b_{m} \lambda_{m}} \log \left(2 \alpha_{m}\left(\frac{2\left|q_{m}(z)\right|}{m^{b_{m}}}\right)^{\lambda_{m}} \frac{1}{m^{b_{m}}\left|q_{m}(z)\right|-2}\right) \\
& <\frac{2}{b_{m} \lambda_{m}} \log \left(2 \alpha_{m}\right)+\frac{2 \log \left(2\left|q_{m}(z)\right|\right)}{b_{m}}-\frac{2}{b_{m} \lambda_{m}} \log \left(\frac{1}{m^{b_{m}}}\right)^{\lambda_{m}} \\
& <3+2 C_{1}-2 \log m<-\log m
\end{aligned}
$$

for $m$ sufficiently large. If $w=f(z)$ with $z \in L_{m}^{\prime}$, from (21) we have

$$
\begin{aligned}
& \frac{2}{b_{m} \lambda_{m}} \log \left|p_{m}(z, f(z))\right| \\
& \leq \frac{2}{b_{m} \lambda_{m}} \log \left(R\left(\frac{4}{m^{b_{m}}}\right)^{2 \lambda_{m}}+\alpha_{m}\left|q_{m}(z)\right|^{\lambda_{m}} \sum_{j=0}^{\lambda_{m}}\left(\frac{4}{m^{b_{m}}}\right)^{j}\left|q_{m}(z)\right|^{\lambda_{m}-j}\right) \\
& \quad<\frac{2}{b_{m} \lambda_{m}} \log \left(R\left(\frac{4}{m^{b_{m}}}\right)^{2 \lambda_{m}}+\left(\frac{4}{m^{b_{m}}}\right)^{\lambda_{m}} \alpha_{m}\left(\frac{4}{m^{b_{m}}}+\frac{4}{m^{b_{m}}}\right)^{\lambda_{m}}\right) \\
& \leq \frac{2}{b_{m} \lambda_{m}} \max \left\{\log \left(2 R\left(\frac{4}{m^{b_{m}}}\right)^{2 \lambda_{m}}\right), \log \left(2 \alpha_{m} 2^{\lambda_{m}}\left(\frac{4}{m^{b_{m}}}\right)^{2 \lambda_{m}}\right)\right\} \\
& \leq \frac{2}{b_{m} \lambda_{m}} \log \left(2 \alpha_{m} 2^{\lambda_{m}}\right)+\frac{2 \log 4}{b_{m}}-4 \log m<-\log m
\end{aligned}
$$

for $m$ sufficiently large. The proof is thereby completed.

\section{References}

[BT] E. Bedford and B. A. Taylor, A new capacity for plurisubharmonic functions, Acta Math. 149 (1982), 1-40. 
[Ch] E. Chirka, Expansions in series and the rate of rational approximations for holomorphic functions with analytic singularities, Mat. Sb. (N.S.) 22 (1974), 323-332.

[EW1] A. Edigarian and J. Wiegerinck, The pluripolar hull of the graph of a holomorphic function with polar singularities, Indiana Univ. Math. J. 52 (2003), 1663-1680.

[EW2] - - - Determination of the pluripolar hulls of graphs of certain holomorphic functions, Ann. Inst. Fourier (Grenoble) 54 (2004), 2085-2104.

[FN] J. E. Fornæss and R. Narasimhan, The Levi problem on complex spaces with singularities, Math. Ann. 248 (1980), 47-72.

[Hö] L. Hörmander, Notions of Convexity, Birkhäuser, 1994.

[Kl] M. Klimek, Pluripotential Theory, Oxford Univ. Press, 1991.

[LNT] Le Mau Hai, Nguyen Quang Dieu and T. V. Long, Remarks on pluripolar hulls, Ann. Polon. Math. 84 (2004), 225-236.

[LP] N. Levenberg and E. Poletsky, Pluripolar hulls, Michigan Math. J. 46 (1999), 151-162.

[N1] Nguyen Quang Dieu, Pluripolar hulls of graphs, preprint, 2004.

[N2] -, Weak Runge pairs in $\mathbb{C}^{n}$, J. Math. Anal. Appl. 327 (2007), 71-78.

[Ra] T. Ransford, Potential Theory in the Complex Plane, Cambridge Univ. Press, 1995.

[Sa] A. Sadullaev, Rational approximations and pluripolar sets, Mat. Sb. (N.S.) 119 (1982), 96-118 (in Russian).

[Sk] H. Skoda, Sous-ensembles analytiques d'ordre fini ou infini dans $\mathbb{C}^{n}$, Bull. Soc. Math. France 100 (1972), 353-408.

[Wi1] J. Wiegerinck, The pluripolar hull of $\left\{w=e^{-1 / z}\right\}$, Ark. Mat. 38 (2000), 201-208.

[Wi2] -, Graphs of holomorphic functions with isolated singularities are complete pluripolar, Michigan Math. J. 47 (2000), 191-197.

[Ze] A. Zeriahi, Ensembles pluripolaires exceptionnels pour la croissance partielle des fonctions holomorphes, Ann. Polon. Math. 50 (1989), 81-89.

Department of Mathematics

Hanoi University of Education (Dai Hoc Su Pham Hanoi)

Cau Giay, Tu Liem, Hanoi, Vietnam

E-mail: dieu_vn@yahoo.com

tangvan.long@gmail.com

Received 15.5.2006

and in final form 22.12.2006 